\title{
Coaliciones electorales y nuevos partidos políticos en Argentina. El caso de Propuesta Republicana
}

\author{
Electoral coalitions and new political parties in \\ Argentina. The Republican Proposal Party case
}

\author{
Sebastián Mauro iD https://orcid.org/0000-0001-8849-6562 \\ Universidad de Buenos Aires, Argentina,smauro@sociales.uba.ar
}

\begin{abstract}
Republican proposal (PRO) is a party founded in 2002 that reached the Presidency of Argentina in 2015. The fact that an extra-bipartisan organization won a national election is an unprecedented phenomenon, which contradicts the predictions of the literature, which focused on analyzing the federalization of party system exclusively in terms of the denationalization of the two Argentine main parties. Which nationalization strategies were followed by PRO? The article proposes that PRO developed what we have called "subnational strategy", based on the opportunities offered by fragmentation and federalization of the Argentine party system. To identify such subnational strategy, the article reconstructs the electoral path of PRO in the 24 districts of the Argentine system for the national and subnational level, describing alliances and candidate nominations. Among key findings, the article identifies the characteristics of the "subnational strategy" and addresses the problems of the diagnoses on the territorial fragmentation of Argentine politics.
\end{abstract}

Key words: electoral coalitions, political parties, Argentina.

Resumen: Propuesta Republicana (PRO) fue fundado en 2002 y conquistó la presidencia de Argentina en 2015. El triunfo de una fuerza extrabipartidista (diferente de radicales y peronistas) es un fenómeno inédito, que contradice las predicciones de la literatura politológica. ¿Qué estrategias desarrolló PRO para nacionalizarse? El artículo propone que PRO desarrolló lo que hemos denominado "estrategia subnacional", que aprovecha las oportunidades que ofrece la fragmentación y federalización del sistema partidario argentino. Para identificar dicha estrategia subnacional, el artículo reconstruye la trayectoria electoral de PRO en los 24 distritos del sistema electoral argentino, para los niveles de cargos nacional y provincial, atendiendo especialmente a la política de alianzas y a la nominación de candidatos.

Recepción:

20/02/2019

Aprobación: 09/01/2020
Entre los principales hallazgos, el artículo identifica las características de la mencionada "estrategia subnacional" y plantea la necesidad de revisar los diagnósticos sobre la fragmentación territorial de la política argentina.

Palabras clave: coaliciones electorales, partidos políticos, Argentina. 


\section{Introducción}

La evolución de Propuesta Republicana (PRO), partido político de reciente creación, constituye un fenómeno inédito en la política argentina contemporánea. Por primera vez desde la transición democrática, una fuerza extrabipartidista ${ }^{1}$ controlaba, a febrero de 2019, la Presidencia de la República (en coalición con la Unión Cívica Radical [UCR] y otros partidos), dos ejecutivos subnacionales y contingentes legislativos a nivel nacional, que nunca registró un partido ajeno al Partido Justicialista (PJ) y la UCR (21\% de las bancas en la Cámara de Diputados y 13\% de las bancas en el Senado). Incluso antes de alcanzar la Presidencia de la República en 2015, PRO ya había logrado resultados electorales inéditos para una fuerza extrabipartidista, al conquistar el gobierno de la Ciudad Autónoma de Buenos Aires en 2007 y una nutrida representación legislativa (entre un tercio y la mitad de las bancas en la Legislatura porteña, según el periodo legislativo).

PRO es un partido de redes (Scherlis, 2009), creado como reacción a la crisis política argentina de 2001, cuando las intensas protestas sociales forzaron la renuncia del presidente Fernando de la Rúa (UCR, aliado al Frente País Solidario [FREPASO]) y de cuatro presidentes provisionales posteriores (tanto radicales como peronistas). Como otras experiencias extrabipartidistas previas, ${ }^{2}$ un liderazgo personalista (en este caso, del empresario y dirigente futbolístico Mauricio Macri) construyó un entramado de relaciones políticas radial y flexible: es decir, que en torno de la figura del líder giran cuadros políticos propios, aliados de otros partidos, consultores políticos y figuras extrapartidarias según las necesidades de la coyuntura (Mauro, 2011 y 2015; Vommaro et al., 2015; Vommaro y Morresi, 2014; Mattina, 2015).

También, al igual que las mencionadas experiencias extrabipartidistas previas, PRO nació y se desarrolló durante una década como un partido fuertemente localizado en la Ciudad Autónoma de Buenos Aires (CABA) y sus alrededores. La CABA fue históricamente el terreno propicio para el

1 Es decir, ajeno a los dos partidos tradicionales: el Partido Justicialista (PJ, o peronismo) y la Unión Cívica Radical (UCR, o radicalismo).

2 Por ejemplo, el FREPASO, fundado en 1994 por el peronista disidente Carlos "Chacho" Álvarez, que llegó al gobierno nacional en 1999 junto con la UCR (Alianza por la Educación y el Trabajo); o el partido Afirmación por una República Igualitaria (ARI), fundado por la radical disidente Elisa Carrió en 2003, que, luego de cambiar su denominación a Coalición Cívica (CC), llegó al gobierno nacional junto con el PRO y la UCR en 2015 (en la alianza Cambiemos). 
crecimiento de nuevas fuerzas políticas, en especial desde el deterioro electoral de la UCR en la década de 1990 (Torre, 2003), y sobre todo luego del colapso del sistema político durante la crisis de 2001 (Bril Mascarenhas, 2007; Mauro, 2011).

Las condiciones favorables que ofrecía la CABA, sin embargo, no se replicaban en el resto de los distritos (provincias), en los cuales la literatura (Calvo y Escolar, 2005; Leiras, 2007; De Luca y Malamud, 2005 y 2016; Malamud, 2011; Escolar y Castro, 2014; Suárez y Pegoraro, 2014) identifica estabilidad en la competencia electoral e incluso una tendencia a la reelección de los oficialismos provinciales. Sobre este último punto se organiza el presente artículo: la trayectoria politica del PRO contradice las predicciones de la literatura politológica acerca de la estabilidad de los sistemas de partidos provinciales y la imposibilidad de la nacionalización de partidos de nueva creación.

La hipótesis común de los estudios publicados en las últimas dos décadas (Calvo y Escolar, 2005; Leiras, 2007; De Luca y Malamud, 2005 y 2016; Malamud, 2011; Escolar y Castro, 2014; Suárez y Pegoraro, 2014), es que la federalización del sistema partidario argentino constituye un obstáculo para la nacionalización de nuevos partidos, por lo tanto una fuerza extrabipartidista solo podría nacionalizarse como aliado menor de un partido tradicional, o quedaría relegada a los centros metropolitanos, los cuales, además, se caracterizan por contar con un electorado tendencialmente menos leal y más volátil que en las provincias periféricas (que representan una proporción baja del electorado nacional).

Sin embargo, Mauricio Macri alcanzó la presidencia en 2015 y es el único líder de una coalición donde un partido tradicional (la UCR) y otros partidos menores (la CC y otros partidos) ocuparon un lugar subordinado. Surge entonces la pregunta: ¿el triunfo de Mauricio Macri en las presidenciales de 2015 fue un fenómeno coyuntural o revela la necesidad de revisar los supuestos sobre el sistema partidario argentino?

El presente trabajo sostiene que PRO ha desarrollado una estrategia de dos tiempos para instalarse y luego expandirse territorialmente, basada en capturar los fragmentos partidarios en disponibilidad, surgidos de la fragmentación de las organizaciones partidarias argentinas. A diferencia de lo que ha postulado la literatura, dicha fragmentación se extiende hacia el interior de las unidades subnacionales, facilitando la formación de coaliciones electorales incongruentes y volátiles. El argumento se dividirá en dos apartados. En el primero presentaré los principales elementos con los que la literatura caracteriza la evolución del sistema partidario argentino luego de 2001, y 
señalaré algunos de sus puntos oscuros. En el segundo apartado analizaré la estrategia de PRO estableciendo dos periodos: la fase de desarrollo de una "estrategia subnacional" y la etapa de nacionalización por efecto arrastre. Finalmente presentaré las conclusiones.

\section{Desalineamiento y federalización del sistema partidario}

El ciclo iniciado en 1983 ha evolucionado de un sistema bipartidista integrado (De Riz, 1992) a un sistema multipartidista federalizado (Escolar y Castro, 2014). Al comienzo del ciclo, casi la totalidad de los cargos electivos se distribuían entre el PJ y la UCR, con participación minoritaria de partidos provinciales y de fuerzas políticas nacionales pero exclusivamente arraigadas en algunas de las grandes ciudades. Desde mediados de la década de 1990, la suma de votos reunidos por los dos principales partidos ha tendido a decrecer en tiempos y magnitudes diferentes según el tipo de cargo (Calvo y Escolar, 2005), el territorio (Scherlis, 2009) y el partido (Torre, 2003), como ilustra la Figura $1 .^{3}$

El retroceso del bipartidismo despertó el interés por los terceros partidos (Abal y Suárez, 2003), pero luego de la crisis de 2001 la literatura se enfocó en la desnacionalización de las organizaciones partidarias. Según esta perspectiva, las élites partidarias subnacionales han desplegado estrategias de control de limites ${ }^{4}$ para protegerse del impacto del desalineamiento de la ciudadanía en elecciones nacionales, utilizando estratégicamente las prerrogativas institucionales que el federalismo les habilita: modificación de reglas electorales provinciales (Calvo y Escolar, 2005), uso partidario del calendario electoral (Oliveros y Scherlis, 2006), creación de etiquetas partidarias locales (Galván, 2011), política de alianzas incongruentes (Clerici, 2014), entre otras.

Esta línea de razonamiento llevó a la conclusión, explicitada por De Luca y Malamud (2005 y 2016) de que la federalización del sistema partidario constituye un obstáculo infranqueable para la expansión de nuevos partidos más allá de los subsistemas partidarios descongelados (es decir, aquellos subsistemas partidarios donde se registraba el desalineamiento del electorado no peronista respecto de la UCR, como por ejemplo en la CABA). Las terceras fuerzas pueden conectarse circunstancialmente con un clima de opinión en los grandes centros urbanos, y desde allí cobrar protagonismo en la arena comunicacional y legislativa, pero difícilmente podrían superar la heterogeneidad

3 Todas las figuras se encuentran en el Anexo, al final del presente artículo (Nota del editor).

4 Tomo el concepto del reconocido trabajo de Gibson (2005), extendiendo la estrategia más allá de los gobiernos subnacionales autoritarios. 
territorial del sistema argentino y, en especial, conquistar la presidencia de la República o los Ejecutivos provinciales.

Con correcciones, los estudios sobre la evolución del sistema partidario argentino han tendido a dar por válida esta caracterización, según la cual el problema del sistema partidario argentino se cifra en la incapacidad de los partidos políticos tradicionales de coordinarse verticalmente (Leiras, 2007). En este sentido, Suárez y Pegoraro (2014) afirman que la federalización del sistema partidario argentino sería consecuencia casi exclusiva de la estrategia de territorialización del peronismo (observada por Gibson y Calvo, 2000; Levitsky, 2005), minimizando la influencia de otros actores.

Esta descripción - fundamentada en un gran volumen de evidencia empírica- adolece de un problema: traslada los supuestos de cohesión partidaria y estabilidad de la competencia electoral de la escala nacional a la escala provincial. Ello condujo a la literatura mencionada a restarle importancia a dos fenómenos que son relevantes para analizar la estrategia de nacionalización de PRO: la multiplicación de etiquetas partidarias y la expansión de la lógica aliancista en los procesos electorales.

Las Figuras 2 y 3 ilustran el crecimiento simultáneo del número de etiquetas partidarias que compiten en elecciones nacionales y de la proporción de estrategias aliancistas en el total de la oferta electoral. La Figura 2 muestra el crecimiento del número de partidos nominales que compitieron por la Presidencia entre 1983 y 2015 ; mientras que en 1983 solo 3\% de los partidos compitió en el marco de alianzas electorales, en 2015 el 99\% de los partidos compitió por la presidencia en el marco de coaliciones.

La Figura 3 ilustra la misma evolución en la competencia por bancas en la Cámara de Diputados de la Nación: en 1983 el 13\% de los partidos compitieron en el marco de alianzas, mientras que en 2015 participó el 83\%.

Según la literatura citada, ambos fenómenos se explican exclusivamente por la baja integración vertical de los partidos tradicionales. No obstante, como lo ilustra la Figura 4, en el nivel subnacional también se ha incrementado significativamente la proporción de partidos politicos que compiten en alianzas electorales.

La literatura indica que los gobernadores son jefes partidarios indiscutidos (Calvo y Micozzi, 2005), que se ha reducido la competitividad en la mayoría de las arenas provinciales (Calvo y Escolar, 2005; Suárez y Pegoraro, 2014) e incluso que en muchos distritos subnacionales imperan regímenes políticos deficitariamente democráticos (Gervasoni, 2011; Gibson, 2005). Sin embargo, la proporción de alianzas sobre el total de la oferta política se ha incrementado en todos los distritos, incluso en aquellos donde ha decrecido 
de forma progresiva la competitividad del sistema, medida por la contracción del Número Efectivo de Partidos (NEP) y por la ampliación de los márgenes por los cuales los oficialismos se reeligen.

La Figura 5 ilustra este fenómeno, comparando la proporción de estrategias aliancistas por sobre el total de la oferta política en dos grupos de sistemas partidarios subnacionales (identificados con el cuartil "más competitivo" y el cuartil "menos competitivo"), durante cinco elecciones legislativas subnacionales. ${ }^{5}$ Los subsistemas con los mayores promedios de NEP, con los mayores niveles de alternancia y con los márgenes de triunfo electoral más acotados siguen el mismo patrón de estrategias aliancistas que las seis provincias con signos de menor competencia y con baja o nula alternancia en el Ejecutivo.

En resumen, los estudios reseñados en este apartado detectan un retroceso del bipartidismo y se lo adjudican a la federalización del sistema partidario, la cual entienden como un déficit de integración vertical de las unidades subnacionales de los partidos políticos, que son supuestamente homogéneas e integradas en cada arena provincial. Sin embargo, como hemos señalado, la multiplicación de las etiquetas partidarias y de las alianzas electorales predomina también a nivel subnacional, e incluso es regla en todos los distritos, independientemente del nivel de competitividad del sistema.

Si la tendencia a la multiplicación de etiquetas partidarias y de alianzas electorales predomina en todas las arenas subnacionales, independientemente del nivel de competitividad de cada sistema, debemos concluir que

5 Las provincias fueron ordenadas según tres indicadores medidos entre 1983 y 2015: i) el promedio del NEP electoral para gobernador, ii) número de partidos en el Ejecutivo, y iii) promedio del índice de competitividad para elecciones ejecutivas. Los seis sistemas seleccionados como menos competitivos son Formosa, La Rioja, Neuquén, San Luis, Santiago del Estero y Santa Cruz. En cinco de estas provincias nunca cambió el partido en el gobierno. En cuatro, los oficialismos se han impuesto por una diferencia promedio de 30 puntos porcentuales durante todo el periodo, y en cinco de estas provincias el NEP según votos para gobernador supera ligeramente los dos partidos efectivos. En esta muestra podrían incorporarse otras provincias que exhiben valores similares en algunos de estos criterios, pero han sido seleccionados estos casos por mostrar valores cercanos al extremo en los tres criterios combinados. En el extremo opuesto, hemos seleccionado a la CABA, Tierra del Fuego, Córdoba, Mendoza, Río Negro y Santa Fe. En cinco de estos casos, el promedio del NEP electorales para gobernador se ubica cerca de los tres partidos. Cuatro de estas provincias muestran una alta competitividad: en las nueve elecciones a gobernador, el promedio de la diferencia entre el partido ganador y el principal contendiente ha sido menor a los 10 puntos porcentuales; mientras que en tres de ellas ha cambiado al menos dos veces el partido de gobierno. 
el retroceso del bipartidismo no se debe a la falta de integración nacional de las organizaciones partidarias subnacionales, y que deben intervenir otras variables explicativas. Si, como sostienen algunos estudios (Aboy Carlés, 2009; Cheresky, 2008; Mauro, 2011), el retroceso del bipartidismo y la federalización del sistema de partidos obedece a una reconfiguración de las identidades políticas, según la cual las etiquetas partidarias no producen identidad en el electorado, antes que a problemas de integración de las organizaciones, entonces debemos esperar una evolución diferente a la esperada por trabajos como los analizados antes. En particular, la tesis, sostenida principalmente por Malamud y de Luca (2005 y 2016), de que un sistema partidario federalizado impide la nacionalización de nuevas fuerzas políticas.

Al contrario, si asistimos a la federalización del sistema político por la incapacidad de las etiquetas tradicionales de producir identidad, es esperable que de la fragmentación territorial de los partidos emerjan oportunidades (y no obstáculos) a la nacionalización de nuevos partidos y líderes, dado que se multiplican los fragmentos de estructuras partidarias en disponibilidad, susceptibles de ser incorporados a distintas coaliciones electorales según la coyuntura.

Precisamente de estos fenómenos se alimentó PRO para constituirse en una fuerza política competitiva a nivel nacional, como analizaremos en el próximo apartado.

\section{El PRO y la estrategia subnacional}

El principal argumento de este artículo consiste en señalar que el PRO advirtió el marco de oportunidades del sistema partidario argentino resultante de la crisis de 2001 e implementó una estrategia de autoconstrucción y expansión territorial en consecuencia. Su estrategia se basaría en: (a) jugar el capital político de su principal referente en elecciones ejecutivas, para (b) atraer fragmentos partidarios en disponibilidad e incorporarlos en una estructura política flexible (coaliciones partidarias que reemplazarían sucesivamente a la denominación del propio partido ${ }^{6}$ ).

6 De este modo, Compromiso para el Cambio era el nombre del partido fundado en 2002 por Macri y al mismo tiempo el nombre de la alianza electoral que lo postuló a la Jefatura de Gobierno de la CABA en 2003 (alianza de la que participaban partidos marginales del sistema político porteño). En 2005, Compromiso para el Cambio se alió al partido RECREAR para conformar la alianza PRO. En 2008, tras la disolución de RECREAR, PRO pasó a ser el nombre del partido (y de la alianza electoral en la CABA). En 2015, PRO se alió a la UCR y a la Coalición Cívica para formar la alianza Cambiemos, que progresivamente reemplazaría al PRO como denominación del macrismo. 
Dicha estrategia se desarrollaría en dos etapas: instalación y consolidación a nivel local (2003-2011), para luego pasar a una rápida nacionalización, traccionada por la candidatura presidencial de Mauricio Macri (2013-2015). Posponer la candidatura presidencial de Macri y anclarse tempranamente en una arena subnacional era una estrategia inexplorada por las terceras fuerzas precedentes, e incluso fue una opción desestimada por otros referentes políticos extrabipartidistas contemporáneos.

\section{Instalación y consolidación en el nivel local (2003-2011)}

Los inicios del emprendimiento político de Mauricio Macri han sido descritos en otros trabajos (Mauro, 2011; Vommaro et al., 2015; Mattina, 2015). El empresario inició su carrera pública en 1995, cuando fue electo para presidir el popular club de futbol Boca Juniors. Durante ese periodo convocó a cuadros técnicos y constituyó una fundación, con el objetivo de prepararse para un eventual salto a la política. La crisis de 2001 aceleró los plazos para dar ese paso, y en 2003 se alió al dirigente peronista Horacio Rodríguez Larreta para promover su candidatura como Jefe de Gobierno de la Ciudad Autónoma de Buenos Aires. A pesar de que podía aspirar a una candidatura competitiva en las elecciones presidenciales, Macri se enfocó en la $\mathrm{CABA}$, al percibir que el distrito atravesaba un periodo de extrema fragmentación política (Bril-Mascarenhas, 2007; Mauro, 2011). Dado que las encuestas le asignaban un potencial competitivo, referentes de fragmentos de partidos políticos se asociaron a su candidatura, especialmente dirigentes del peronismo local y otros provenientes de los partidos minoritarios de centro-derecha del distrito.

El partido fundado por Macri (Compromiso para el Cambio) conquistó $37 \%$ de los votos en el distrito, sin lograr conquistar el Ejecutivo local. ${ }^{7}$ Sin embargo, su coalición conquistó cinco bancas en la Cámara de Diputados de la Nación y 38\% de las bancas en la Legislatura local (23 sobre 60). Sin enfrentar los costos de construir una candidatura presidencial ${ }^{8}$ (que hubiera

7 Datos del Tribunal Superior de Justicia de CABA. Los datos referidos a escaños fueron extraídos de la Honorable Cámara de Diputados de la Nación y de la Legislatura de la CABA.

8 El ex referente radical Ricardo López Murphy, por ejemplo, había fundado el partido RECREAR y en 2003 obtuvo 16\% de los votos en las presidenciales. Dadas las características del sistema electoral argentino y las particularidades del ciclo electoral de 2003 (donde se disociaron las fechas de los comicios ejecutivos y legislativos nacionales), solo conquistó una banca en la Cámara de Diputados, en representación de la CABA. Un caso parecido fue el de la diputada Elisa Carrió, quien fundó su propio partido (ARI). 
exigido sumar apoyos en los distintos territorios subnacionales), Macri se instaló como referente político con apoyo electoral y una cuota de poder institucional en CABA.

Mauricio Macri debió esperar al siguiente turno (2007) para alcanzar el gobierno de la CABA, luego de que el jefe de Gobierno en ejercicio fuera destituido mediante Juicio Político y la coalición oficialista local se fracturara (Mauro, 2011).

El control del gobierno porteño garantizó la cohesión interna del macrismo. Como ha señalado Scherlis (2009), los partidos de redes, desanclados de pertenencias estables en la sociedad civil, solo pueden desarrollarse en el marco de la esfera estatal. Considerando, además, que la CABA es uno de los distritos más ricos del país, PRO estaba en condiciones de distribuir beneficios para atraer a nuevos círculos de aliados, y al mismo tiempo podía exhibir logros de gestión atractivos para los electorados de los otros distritos. ${ }^{9}$ El control del gobierno, asimismo, garantizaba la permanencia de la figura de Macri entre los principales referentes del país, sin involucrarse cotidianamente en el debate interpartidario.

Resulta claro, entonces, que el control del Ejecutivo porteño era un capital político fundamental para PRO, pero también lo era la relativamente buena imagen de Mauricio Macri. En este sentido, la expansión de PRO podía seguir dos cursos divergentes. El primero, jugar el liderazgo de Macri en elecciones presidenciales (explotando una estrategia de nacionalización vertical, según el modelo de Albala et al., 2017), corriendo el riesgo de perder el Ejecutivo local a manos de un opositor o de un sucesor potencialmente desleal. El segundo, resignar la expansión territorial apostando a la reelección en el gobierno local.

Estudios previos (Mauro, 2015; Mattina, 2012; Vommaro y Morresi, 2014) han reconstruido en detalle las coyunturas críticas en las cuales el macrismo se vio en la obligación de decidir entre estas dos alternativas. Mauricio Macri evitó postularse a la presidencia, y solo lo hizo cuando ya no pudo reelegirse en la Jefatura de Gobierno porteña, lo que accidentalmente coincidió con una coyuntura particular, producida también por la imposibilidad de postular a Cristina Fernández para un tercer mandato (Mauro, 2017).

Hasta que no postuló a Mauricio Macri a la presidencia, PRO optó por consolidarse en la arena de la CABA y recurrió a distintos mecanismos para

9 Como señala Vommaro (2017), PRO publicitó en las provincias el estilo de vida porteño, construyendo su gestión en una verdadera marca (brand). Iniciativas modestas, como la delimitación de carriles viales exclusivos para la circulación de buses, fueron elevadas al rango de un modelo de gestión de lo público. 
penetrar en otras arenas provinciales, o al menos para establecer una mínima estructura formal que sirviera a la futura candidatura presidencial de Macri. El principal recurso que utilizó el PRO para su expansión territorial fue la celebración de alianzas electorales con distintos partidos opositores al kirchnerismo en las provincias. Las alianzas celebradas por PRO durante el periodo 2003-2011 fueron marcadamente incongruentes ${ }^{10}$ (variables según el distrito y el tipo de cargo en disputa) y volátiles (variables en el tiempo).

En primer lugar, PRO -como partido nacional- no postuló candidato presidencial propio ni participó de alianzas nacionales para la presidencia en 2007 ni en 2011. Las filiales de distrito sí compitieron por bancas en la Cámara de Diputados en el marco de alianzas con distintos partidos, algunas de las cuales, en varias provincias, apoyaron alguna candidatura presidencial. Podría decirse que PRO procuró mantener la autonomía respecto de otros partidos nacionales, e incluso buscó erosionar las oportunidades electorales de otros referentes opositores (Mauro, 2015). En este sentido, el caso saliente fue la candidatura de Ricardo López Murphy (RECREAR) en 2007, a la cual el PRO porteño apoyó tímidamente y solo se concretó una alianza en cinco distritos. ${ }^{11}$ La candidatura presidencial fracasó (obtuvo menos de $2 \%$ de los votos), y pasadas las elecciones, el macrismo incentivó a una facción interna de RECREAR (liderada por Esteban Bullrich) para disputar la conducción partidaria. Un año más tarde, López Murphy fue expulsado del partido que fundó, RECREAR se disolvió y Bullrich fue designado ministro de educación en la CABA. En 2011 reincidiría, apoyando alternativamente (según la provincia) la candidatura presidencial de los peronistas disidentes Eduardo Duhalde o Alberto Rodríguez Saá (incluso en muchas provincias presentó candidatos a diputados nacionales sin apoyar a ningún candidato presidencial).

En segundo lugar, como hemos mencionado, el PRO ensayó alianzas electorales para capturar estructuras políticas disponibles en las distintas

10 Sobre el concepto de congruencia aliancista y las formas de medirlo, véase Clerici (2015a).

11 La coalición PRO (CPC-RECREAR) se presentó en la CABA, Córdoba, San Juan, Santa Fe y Santiago del Estero. La lista PRO conquistó solo dos escaños por la ciudad de Buenos Aires, mientras que su performance electoral en los otros tres distritos osciló entre 0.96 y $1.42 \%$. El macrismo enfrentó a RECREAR en Corrientes y nada menos que en la Provincia de Buenos Aires, donde se alió al peronismo disidente para disputar bancas nacionales y cargos provinciales, obteniendo alrededor de $15 \%$ de los votos según el cargo. El macrismo, además, participó de alianzas electorales con el peronismo disidente en Misiones y Tierra del Fuego, con magros resultados. 
provincias. Lentamente se constituyó como partido en las arenas subnacionales y participó de elecciones nacionales y provinciales (véase Figura 6), casi exclusivamente en el marco de alianzas. ${ }^{12}$ Los socios mayoritarios en estas alianzas fueron los dirigentes peronistas opuestos a la conducción nacional partidaria, ejercida de manera informal por Cristina Fernández. ${ }^{13}$

En casi todos los casos, las "coaliciones" consistían en que la etiqueta PRO participara de las listas que postulaban las agrupaciones peronistas disidentes, sin que PRO realmente impusiera candidatos en ningún nivel. La Figura 7 ilustra este punto: las alianzas de las que participó obtuvieron numerosos escaños, pero solo la mitad (principalmente los candidatos de la CABA y algunos de Buenos Aires) se integró al bloque PRO en la Cámara de Diputados, mientras que el resto se sumó a los bloques de las distintas vertientes del peronismo antikirchnerista.

Mientras Mauricio Macri se mantuvo en la arena porteña, PRO no demostró interés en desarrollar estructuras partidarias arraigadas en las provincias. Solo en coyunturas donde podía preverse que el líder de PRO iba a decidirse por dar la batalla nacional (como en 2009 o 2013), el partido intentó instalar candidatos competitivos en algunas arenas provinciales. En esos casos, recurrió principalmente a figuras extrapartidarias, emergentes del ciclo de protestas sociales contra el gobierno nacional en 2008 por la modificación de los impuestos a las exportaciones de productos agrícolas ${ }^{14} \mathrm{O}$ pertenecientes al mundo del deporte o del entretenimiento. El recurso a candidatos extrapartidarios resolvía el problema de la debilidad organizacional y al mismo tiempo lo reforzaba, habida cuenta de que estas individualidades no eran portadoras de liderazgo partidario interno.

En aquellos casos donde las autoridades partidarias provinciales lograban efectivamente arraigarse y pretendían participar de la definición de la política de alianzas o de candidaturas, el PRO nacional resolvía la intervención del partido y el reemplazo de sus referentes (tal como sucedió en ocho distritos). En casi todos estos casos ni siquiera se había consolidado como una fuerza política competitiva o disponía de liderazgos partidarios fuertes. PRO necesitaba construir alianzas para compensar su débil estructura organizativa en el interior, pero al mismo tiempo necesitaba mantener esa estructura débil

12 Por distintas razones (cambios repentinos de alianzas, definiciones locales, etcétera), PRO compitió sin aliados en diez ocasiones. En todas fue completamente marginal.

13 Para una descripción sobre las facciones peronistas disidentes durante el periodo, véase Galván (2011).

14 Para un detalle del denominado "conflicto del campo" entre el gobierno de Cristina Fernández y las entidades de productores agropecuarios, véase Aronskind y Vommaro (2010). 
para poder definir con autonomía golpes de timón en la estrategia aliancista. En este sentido, PRO no solo mudó su estrategia de aliarse con peronistas a aliarse con radicales. Incluso antes de girar hacia las alianzas con la UCR (en 2015), PRO había cambiado de aliados al menos una vez en casi todos los distritos donde había participado (todos excepto CABA, Mendoza, San Luis, Misiones y Tucumán).

\section{Nacionalización de PRO: la candidatura presidencial de Macri}

La estrategia de construir alianzas con los sectores del peronismo disidente llegó a su fin luego de las elecciones legislativas de 2013, cuando el denominado "peronismo disidente" se unificó detrás de la figura de Sergio Massa, dirigente político de la Provincia de Buenos Aires que se postularía a la presidencia en 2015. Sin posibilidades de aliarse con el peronismo, PRO se vio obligado a recurrir a otra estructura partidaria nacional con arraigo territorial. Con la candidatura presidencial de Mauricio Macri ya instalada, encontró esa estructura en la UCR.

El radicalismo llevaba más de una década sin poder instalar un candidato presidencial competitivo, sobreviviendo sobre la base de una estrategia de nacionalización horizontal (Albala et al., 2017), pero sufriendo un constante proceso de fragmentación y erosión. La posibilidad de aliarse con un candidato presidencial competitivo pero sin estructura política nacional parecía una oportunidad única para recuperar capital político en las arenas provinciales mediante el efecto arrastre (coattail effect) ${ }^{15}$ En este sentido, la lectura del radicalismo era idéntica al diagnóstico académico reseñado en el primer apartado: ${ }^{16}$ un liderazgo de opinión arraigado en los centros urbanos solo puede subordinarse a una estructura política nacional si quiere competir por la presidencia.

Lo que finalmente sucedió fue muy diferente: PRO sumó a la UCR como un anillo más en su estructura flexible de alianzas (que pasó a llamarse Cambiemos), y no se resignó a cederle al radicalismo el efecto arrastre de la candidatura presidencial, sino que, en la medida de sus posibilidades, postuló candidatos para los distintos niveles de cargos. Como lo muestra la Figura 7, la mayor parte de las bancas de diputados nacionales conquistadas por la

15 "La fórmula para 2015 parece clara: el radicalismo pone la estructura y se lleva los cargos legislativos y provinciales, Macri pone la intención de voto y quizás se lleve la presidencia. La diferencia es que, si falla, en 2019 seguirá habiendo UCR pero no PRO" (Malamud, 2015).

16 Por ejemplo, este diagnóstico puede encontrarse, con matices, en el análisis que Varetto y del Cogliano (2016) hacen del proceso electoral de 2015. 
alianza Cambiemos fueron para candidatos del PRO y no de la UCR (algo que no sucedía en el esquema de alianzas con el peronismo).

El arraigo territorial de la UCR tampoco significó un obstáculo para que $\mathrm{PRO}$ postulara candidatos a gobernaciones en las provincias donde era competitivo. Por ejemplo, en Misiones, Santa Fe y la CABA ambos partidos compitieron entre sí, mientras que en Buenos Aires, San Juan y Entre Ríos la coalición postuló a candidatos macristas a la gobernación. En otros distritos las candidaturas debieron consensuarse, y Mauricio Macri tuvo la palabra final sobre los acuerdos interpartidarios, sin que la UCR pudiera oponer eficazmente su peso territorial en la mesa de negociación.

Luego de imponerse en las presidenciales, el desequilibrio interno en la alianza Cambiemos fue más evidente. Macri, que ya había definido de manera autónoma como compañera de fórmula a otra referente de PRO, definió casi sin ninguna limitación los funcionarios de su gabinete, ${ }^{17}$ e incluso privilegió la designación de amigos personales u hombres fuertes de negocios antes que a sus aliados. Otro tanto sucedió en las Cámaras de senadores y de diputados, cuyas autoridades legislativas pertenecen a PRO antes que a los otros aliados de Cambiemos. En las elecciones de 2017, si bien puede observarse una distribución más equitativa de las candidaturas para diputados nacionales (véase Figura 7), la campaña fue definida casi exclusivamente por el presidente y su círculo de colaboradores cercanos, de igual modo que la definición en todas las coyunturas críticas que atravesó el gobierno.

\section{Conclusiones}

En las páginas precedentes se ha descrito la trayectoria política y electoral de PRO como resultante de una estrategia deliberada de instalación y nacionalización de una fuerza política extrabipartidista. Ello exigió reconstruir las decisiones tomadas por el partido para encarar ciclos electorales nacionales y provinciales, definiendo candidaturas y políticas de alianzas. Nuestro planteo consiste en señalar una estrategia en dos pasos, privilegiando primero la instalación en un distrito subnacional, para luego apostar a la nacionalización vertical por el efecto arrastre de un candidato presidencial competitivo. Para hacer eso, PRO se valió de la existencia de estructuras partidarias en disponibilidad, tanto en el polo peronista como en el polo no peronista del arco político, y de las ventajas que ofrecen las instituciones electorales argentinas para celebrar alianzas electorales en las distintas arenas

17 En este punto no se diferencia de la tradición de los presidencialismos latinoamericanos, tal como lo ha identificado Corrales (2010). 
provinciales, sin necesidad de construir primero una organización partidaria competitiva en cada distrito.

Más allá de que el éxito del macrismo en su carrera a la Presidencia se debió, en parte, a circunstancias contingentes, poner el foco en reconstruir dicha estrategia de autoconstrucción nos obliga a revisar los supuestos con que la literatura politológica ha analizado el proceso de federalización durante los últimos 15 años. En este sentido, el artículo ha señalado que la federalización del sistema partidario argentino opera como un factor que amplía los márgenes de incertidumbre sobre la dinámica política, antes que reducirlos.

\section{Referencias}

Abal, Juan Manuel y Suárez, Julieta (2003), “Más allá del bipartidismo. El sistema argentino de partidos”, en Revista Iberoamericana, núm. 9, Estados Unidos: University of Pittsburgh Press.

Aboy Carlés, Gerardo (2009). “La sangre de Esteno. Transformaciones de la ciudadanía en la Argentina: del populismo a la inflexión particularista”, en Delamata, Gabriela [comp.]. Movilizaciones sociales: ¿nuevas ciudadanias?, Argentina: Biblos.

Albala, Adrián et al. (2017), "Pathways to Nationalization in Multilevel Presidential Systems: Accounting for Party Strategies in Brazil and Argentina”, en Revista Publius, vol. 47, núm. 4, Reino Unido: Oxford University Press.

Aronskind, Ricardo y Vommaro, Gabriel (2010), Campos de batalla. Las rutas, los medios y las plazas en el nuevo conflicto agrario, Argentina: Prometeo.

Bril-Mascarenhas, Tomás (2007), "El colapso del sistema partidario de la ciudad de Buenos Aires. Una herencia de la crisis argentina de 2001-2002”, en Desarrollo Económico, vol. 47, núm. 187, Argentina: Instituto de Desarrollo Económico y Social.

Calvo, Ernesto y Escolar, Marcelo (2005), La nueva política de partidos en la Argentina. Crisis politica, realineamientos partidarios y reforma electoral, Argentina: Prometeo.

Calvo, Ernesto y Micozzi, Juan Pablo (2005), “The Governor' Backyard: A Seat-Vote Model of Electoral Reform for Subnational Multiparty Races", en The Journal of Politics, vol. 67, núm. 4, Estados Unidos: University of Chicago Press.

Cheresky, Isidoro (2008), Poder presidencial, opinión pública y exclusión social, Argentina: Ediciones Manantial.

Clerici, Paula (2014), "Aliados y Contendientes. Dimensionando el Fenómeno de la Congruencia Aliancista en Argentina (1983-2013)”, [tesis], Argentina: Universidad Torcuato Di Tella.

Clerici, Paula (2015a), "La Congruencia Aliancista de los Partidos Argentinos en Elecciones Concurrentes (1983-2011)”, en Revista Estudios Políticos, núm. 36, España: Centro de Estudios Políticos y Constitucionales.

Clerici, Paula (2015b), "La Creciente Importancia de las Alianzas Electorales en un Escenario de Competencia Territorializada” en Revista SAAP, núm. 9, Argentina: Sociedad Argentina de Análisis Político.

Corrales, Javier (2010), Presidentes sin partido. La politica de las reformas económicas en Argentina y Venezuela en los años 90, Argentina: Siglo XXI. 
De Luca, Miguel y Malamud, Andrés (2005), “The anchor of continuity: party system stability in Argentina, 1983-2003", ponencia presentada en el Congreso European Consortium for Political Research (ECPR), España.

De Luca, Miguel y Malamud, Andrés (2016), “¿Todo sigue igual que ayer? Continuidad y ruptura en el sistema de partidos argentino (1983-2015)", en Freidenberg, Flavia [comp.], Los sistemas de partidos en América Latina (1978-2015). Cono Sur y Paises Andinos, Mexico: Universidad Nacional Autónoma de México.

De Riz, Liliana (1992), "El debate sobre la reforma electoral en la Argentina”, en Desarrollo Económico, vol. 32, núm. 126, Argentina: Instituto de Desarrollo Económico y Social.

Del Cogliano, Natalia y Varetto, Carlos (2016), "Las elecciones subnacionales de 2015 en Argentina: estabilidad con cambio”, en Revista Uruguaya de Ciencia Politica, vol. 25, núm. 1, Uruguay: Universidad de la República.

Escolar, Marcelo y Castro, Luis (2014), "Nacionalización y equilibrio democrático en Estados Multinivel presidencialistas. Los casos de Argentina y Brasil en perspectiva comparada”, en Documento de Trabajo, núm. 21, España: Universidad de Salamanca.

Galván, Facundo (2011), "El sello importa? El rol partidario de los ejecutivos nacionales y provinciales a la luz de las listas peronistas disidentes (1989-2009)", en Revista Postdata, vol. 16, núm. 1, Argentina: PostData.

Gervasoni, Carlos (2011), "Democracia, autoritarismo e hibridez en las provincias argentinas: la medición y causas de los regímenes subnacionales”, en Journal of Democracy en español, núm. 3, Chile: Universidad Católica de Chile.

Gibson, Edward (2005), "Boundary Control. Subnational Authoritarianism in Democratic Countries”, en Revista World Politics, vol. 58, núm. 1, Reino Unido: Cambridge University Press.

Gibson, Edward y Calvo, Ernesto (2000), "Federalism and low-maintenance constituencies: Territorial dimensions of economic reform in Argentina", en Studies in Comparative and International Development, vol. 35, núm. 3, Estados Unidos: Springer.

Leiras, Marcelo (2007), Todos los caballos del rey. La integración de los partidos politicos y el gobierno democrático en la Argentina, 1995-2003, Argentina: Prometeo.

Levitsky, Steven (2005), La transformación del justicialismo. Del partido sindical al partido clientelista. 1983-1999, Argentina: Siglo XXI.

Malamud, Andrés (2011), "Ni mucho gobierno de la opinión ni tanto regreso de la voluntad: bipartidismo recargado”, en Malamud, Andrés y De Luca, Miguel [comps.], La política en tiempos de los Kirchner, Argentina: Eudeba.

Malamud, Andrés (2015), "Lección radical: hay partido para rato", en Clarín, Argentina. Disponible en: https://www.clarin.com/opinion/partido_radical-peronismo-promauricio_macri-ernesto_sanz_0_B17L4fqwQx.html [6 de noviembre de 2018].

Mattina, Gabriela (2012), “Transformaciones de los formatos partidarios en la democracia argentina: una mirada al PRO desde el ciclo electoral 2011”, en Annunziata, Rocío y Cheresky, Isidoro [comps.], Sin programa, sin promesa, Argentina: Prometeo.

Mattina, Gabriela (2015), “De 'Macri' a 'Mauricio'. Una aproximación a los mecanismos de constitución pública del liderazgo político en la Argentina contemporánea”, en Vommaro, Gabriel y Morresi, Sergio [comps.], Hagamos equipo. PRO y la construcción de la nueva derecha en Argentina, Argentina: Universidad Nacional de General Sarmiento. 
Convergencia Revista de Ciencias Sociales, vol. 27, 2020, Universidad Autónoma del Estado de México

Mauro, Sebastián (2011), "Representación e identificaciones políticas en tiempos de solidaridades inestables (Ciudad Autónoma de Buenos Aires, 2001-2007)” [tesis], Argentina: Universidad de Buenos Aires.

Mauro, Sebastián (2015), “El imperativo estratárquico y los actores extrabipartidistas. Los casos del PRO y del PS (2003-2013)”, en Mauro, Sebastián y Ortiz de Rozas, Victoria [comps.], Política Subnacional en Argentina. Enfoques y problemas, Argentina: Universidad de Buenos Aires.

Mauro, Sebastián (2017), "Las elecciones presidenciales y el reagrupamiento de los actores políticos”, en Mauro, Sebastián y Lenarduzzi, Julieta [comps.], La venganza de los buérfanos. Las elecciones nacionales y subnacionales de 2015 en Argentina, Argentina: Universidad de Buenos Aires.

Oliveros, Virginia y Scherlis, Gerardo (2006), "Reformas políticas: Internas abiertas obligatorias y unificación electoral en las elecciones de 2005”, en Cheresky, Isidoro [comp.], La politica después de los partidos, Argentina: Prometeo.

Scherlis, Gerardo (2009), "El partido estatal estratárquico de redes. Apuntes sobre organización política en la era de los partidos no representativos", en Cheresky, Isidoro [comp.], Las urnas y la desconfianza ciudadana en la democracia argentina, Argentina: Homo Sapiens.

Suárez, Julieta y Pegoraro, Mara (2014), “La construcción de un predominio nacional: un análisis de la historia reciente del sistema de partidos multinivel en Argentina (19832011)", en Freidenberg, Flavia y Suárez, Julieta [comps.], Territorio y Poder: Nuevos actores y competencia politica en los sistemas de partidos multinivel en América Latina, España: Universidad de Salamanca.

Torre, Juan Carlos (2003), "Los huérfanos de la política de partidos. Sobre los alcances y la naturaleza de la crisis de representación partidaria”, en Desarrollo Económico, vol. 42, núm. 168, Argentina: Instituto de Desarrollo Económico y Social.

Vommaro, Gabriel y Morresi, Sergio (2014), "Unidos y diversificados: la construcción del partido PRO en la CABA”, en Revista SAAP, vol. 8, núm. 2, Argentina: Sociedad Argentina de Análisis Político.

Vommaro, Gabriel et al. (2015), Mundo PRO: Anatomía de un partido fabricado para ganar, Argentina: Planeta.

Vommaro, Gabriel (2017), La larga marcha de Cambiemos: la construcción silenciosa de un proyecto de poder, Argentina: Siglo XXI.

\section{Otras fuentes consultadas}

Atlas Electoral de Andy Tow, Argentina. Disponible en: https://www.andytow.com/access/ index.php?logout=true [noviembre de 2018].

Cámara Nacional Electoral, Argentina.

Dirección Nacional Electoral, Ministerio del Interior de la Nación, Argentina.

Honorable Cámara de Diputados de la Nación, Argentina.

Legislatura de la Ciudad Autónoma de Buenos Aires, Argentina.

Tribunal Superior de Justicia de Buenos Aires, Argentina.

Tribunales Electorales de las provincias argentinas: Buenos Aires, Catamarca, Córdoba, Corrientes, Chaco, Entre Ríos, Formosa, Jujuy, La Rioja, La Pampa, Mendoza, Misiones, Río Negro, Neuquén, Chubut, Salta, San Juan, San Luis, Santa Cruz, Santa Fe, Santiago del Estero, Tierra del Fuego, Tucumán. 


\section{Anexo}

\section{Figura 1}

\section{Concentración bipartidista en votos y bancas (1983-2015)}

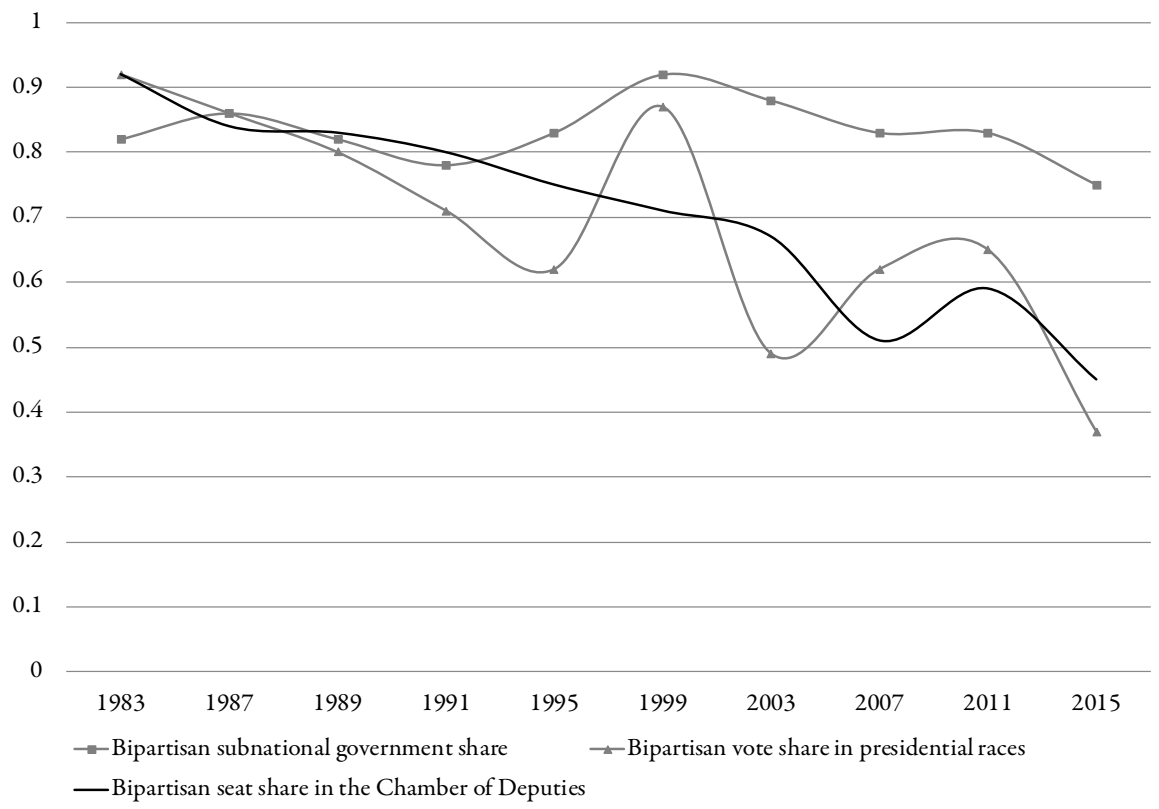

Fuente: Elaboración propia con información de la Dirección Nacional Electoral y la Cámara de Diputados de la Nación.

* En negro: proporción de votos positivos al PJ y a la UCR en elecciones presidenciales. En gris oscuro: promedio de la proporción de bancas en la Cámara de Diputados ocupadas por los bloques del PJ (el bloque que responde a la conducción nacional del partido, en caso de división interna) y la UCR. En gris claro: proporción de votos positivos al PJ y a la UCR en elecciones a Ejecutivos subnacionales.

Nota 1: Para las presidenciales de 2003, se consigna la suma de la proporción de votos obtenidos por los tres candidatos justicialistas, que se presentaron bajo otras etiquetas electorales, de acuerdo con la literatura.

Nota 2: Para las presidenciales de 2015, no se computan votos por la UCR. La UCR no presentó candidatos propios a la presidencia en las elecciones generales, dado que sus precandidatos perdieron las primarias de la alianza Cambiemos con 3\% de los votos. 


\section{Figura 2}

Número de partidos que compiten en elecciones presidenciales, solos o en alianza (1983-2015)

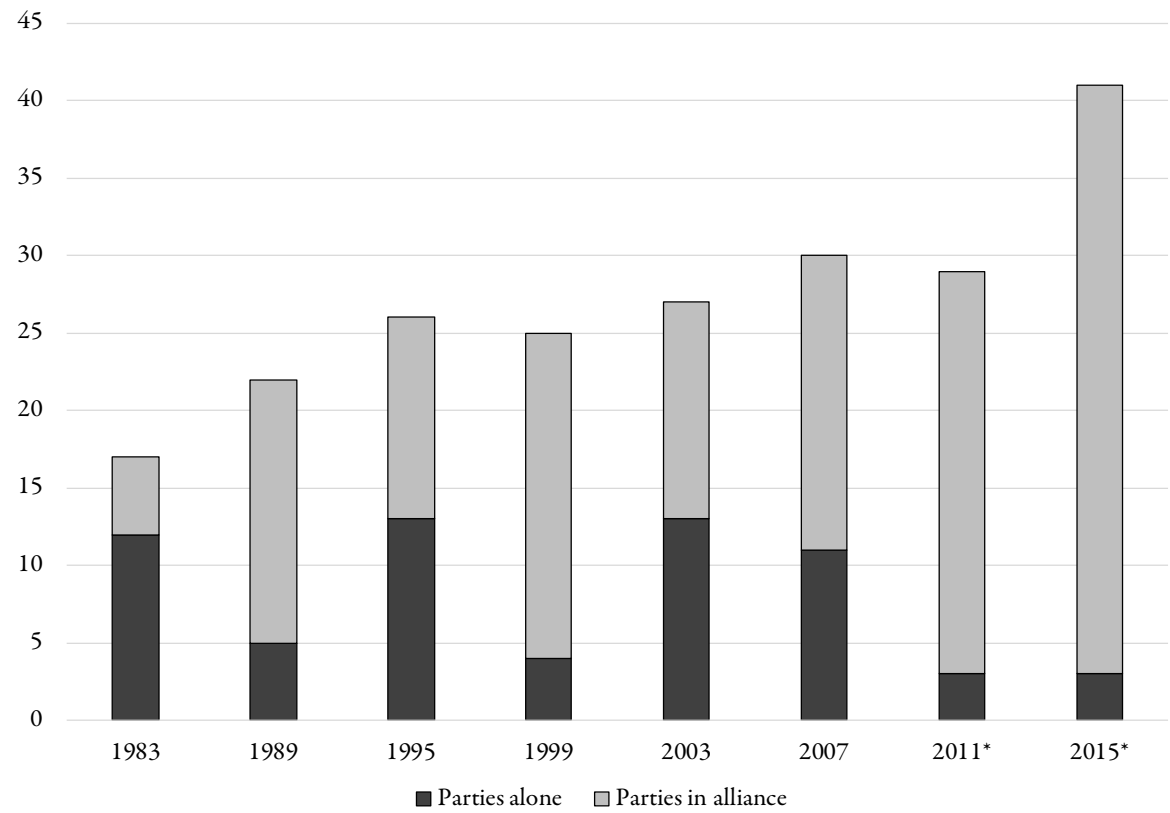

Fuente: Elaboración propia con datos de Clerici (2015a) y de la Cámara Nacional Electoral

* Se consignan datos de las primarias abiertas, simultáneas y obligatorias (PASO). 
Figura 3

Número de partidos que compiten por bancas de diputados nacionales, solos o en alianza (1983-2017)

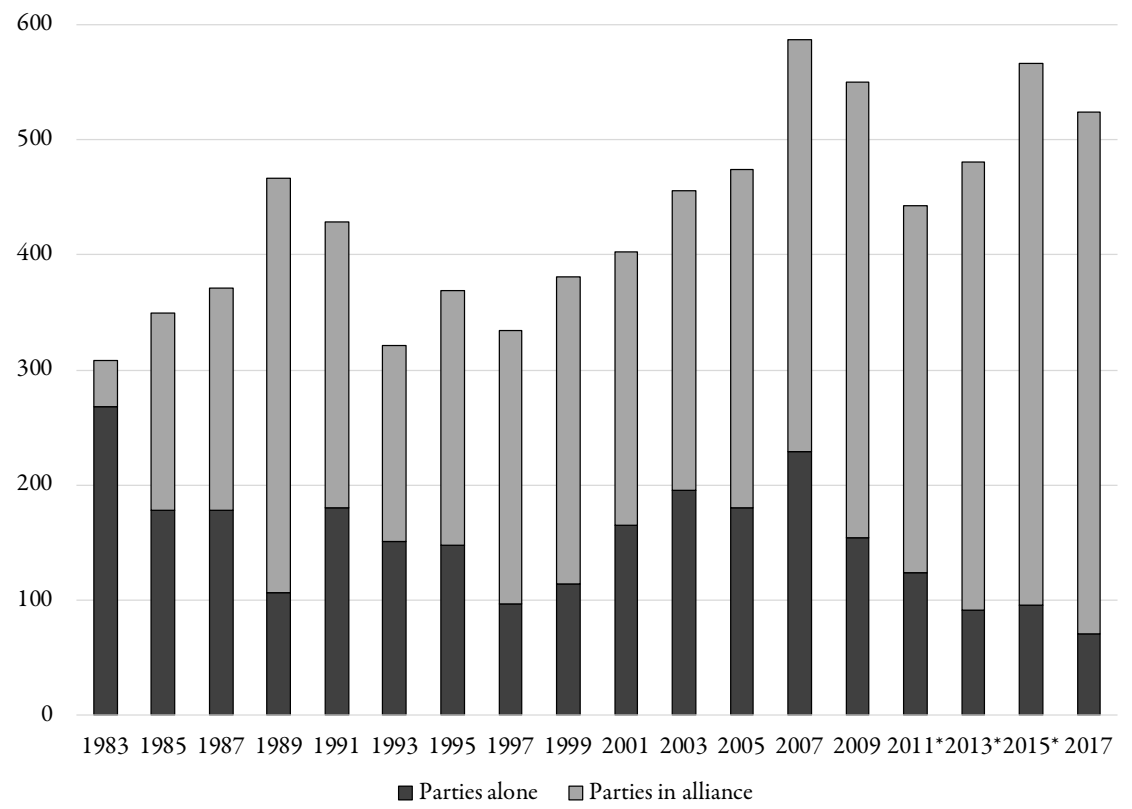

Fuente: Elaboración propia con datos de Clerici (2015a) y de la Cámara Nacional Electoral.

${ }^{*}$ Datos tomados de las PASO. 
Figura 4

Promedio del número de partidos que compiten en elecciones por diputados provinciales, solos o en alianza (1983-2015)

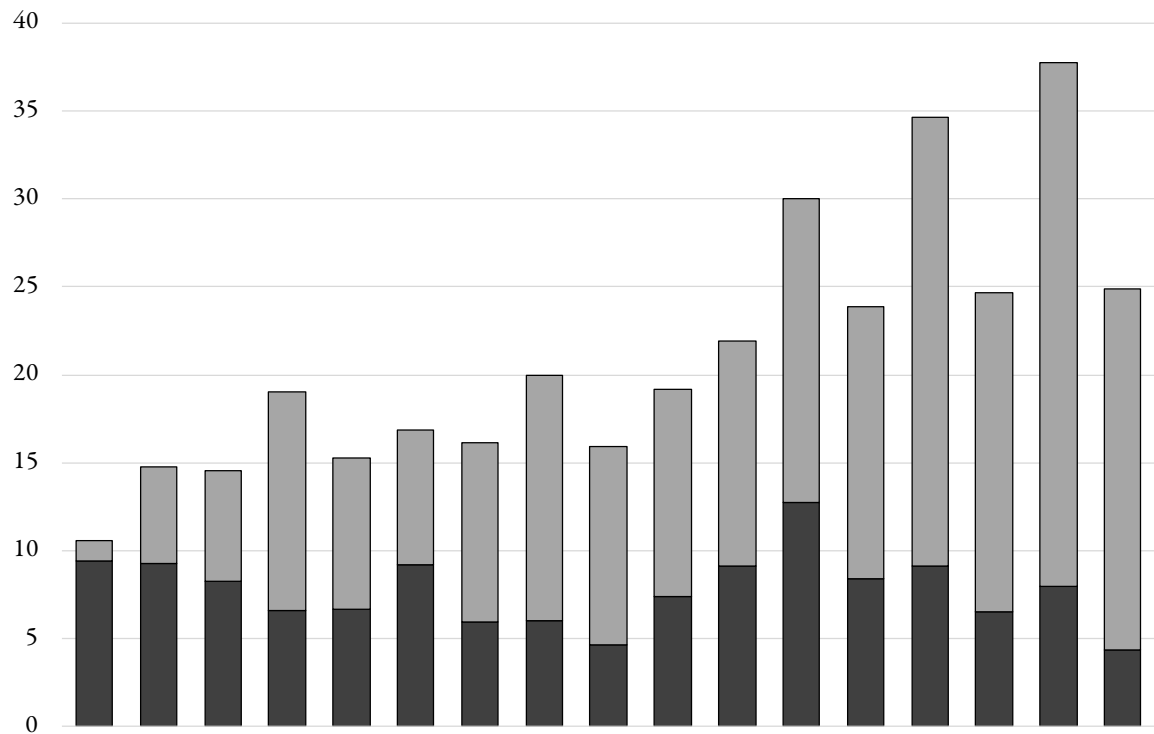

$198319851987198919911993199519971999200120032005200720092011^{*} 2013^{*} 2015^{*}$

$\square$ Average of parties alone in each subnational unit $\square$ Average of parties in alliance in each subnational unit

Fuente: Elaboración propia con datos de Clerici (2015a) y de la Cámara Nacional Electoral.

* Datos tomados de las PASO. 
Sebastián Mauro. Coaliciones electorales y nuevos partidos politicos en Argentina.

El caso de Propuesta Republicana

Figura 5

Media de la proporción de partidos que compiten en el marco de alianzas sobre el total de la oferta política en elecciones legislativas subnacionales, en dos grupos de distritos

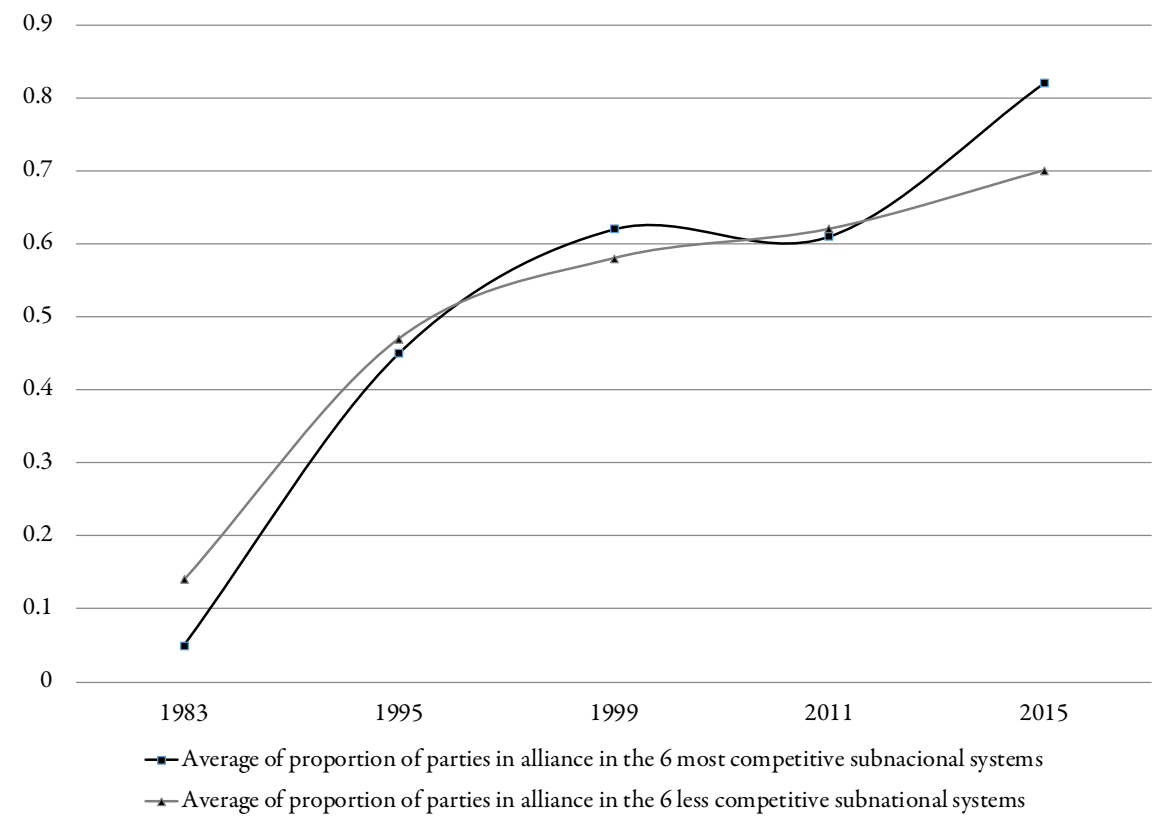

Fuente: Elaboración propia con datos de los tribunales electorales provinciales, de la Dirección Nacional Electoral, del Atlas Electoral de Andy Tow y del Observatorio Electoral Argentino del Centro de Implementación de Políticas Públicas para la Equidad y el Crecimiento (CIPPEC). 
Figura 6

Proporción de distritos en los que el PRO compitió por cargos nacionales y provinciales

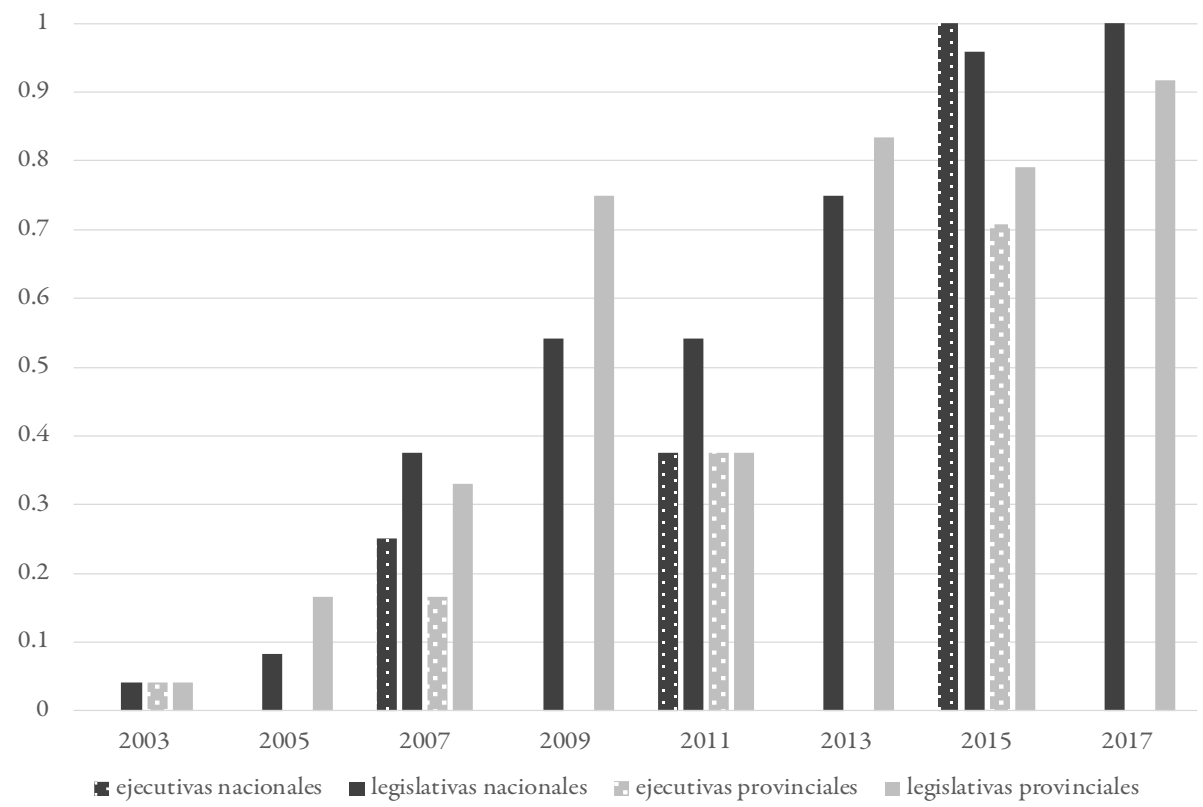

Fuente: Cámara Nacional Electoral y Tribunales electorales provinciales.

Nota 1: La mitad de los distritos elige legisladores provinciales cada cuatro años, por lo que el $\mathrm{N}$ de la variable "legislativas provinciales" es de 24 o de 12, según el caso. Esta variación explica el extraño comportamiento de la variable en 2011: en realidad el PRO compitió en nueve distritos tanto en 2009 como en 2011, pero en el primer caso representa una proporción de 0.75 y en otro de 0.375 .

Nota 2: PRO, como partido nacional, solo participó en elecciones presidenciales en 2015. Sin embargo, las listas de candidatos a diputados nacionales donde participaba PRO fueron adheridas a candidaturas presidenciales en seis distritos en 2007 y en nueve distritos en 2011. 
Sebastián Mauro. Coaliciones electorales y nuevos partidos politicos en Argentina.

El caso de Propuesta Republicana

Figura 7

Distribución de las bancas de diputados nacionales obtenidas por las alianzas electorales en las que participó el PRO (2003-2017)

70

60

50

40

30

20

10

0

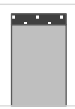

2005

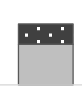

2007

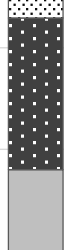

2009

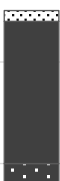

2013

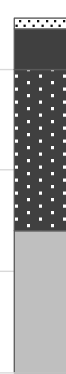

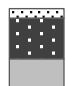

2011 $\square$ Bancas de candidatos del PJ disidente

$\square$ Bancas de candidatos de partidos provinciales

Fuente: Cámara Nacional Electoral y Honorable Cámara de Diputados de la Nación. 
Sebastián Mauro. Doctor en Ciencias Sociales y Licenciado en Ciencia Política por la UBA. Se desempeña como Investigador Adjunto en el Consejo Nacional de Investigaciones Científicas y Técnicas y como Profesor Adjunto en la Universidad de Buenos Aires. Principales líneas de investigación: identidades políticas, partidos políticos y política subnacional. Publicaciones recientes: Mauro, Sebastián, Ortiz de Rozas, Victoria y Paratz, Martín [comps.] (2016), Politica Subnacional en Argentina. Enfoques y problemas, Argentina: Universidad de Buenos Aires; Mauro, Sebastián (2017), "Coalition Politics in a federalized party system. The case of Argentina", en Albala, Adrián y Reniú, Josep [eds.], Coalition Politics and Federalism, Estados Unidos: Springer; Mauro, Sebastián y Rossi, Federico (2015), “The Movement of Popular and Neighborhood Assemblies in the City of Buenos Aires", en Latin American Perspectives, vol. 42, núm. 2, Estados Unidos: Sage. 